\title{
ACCELERATION OF A TWO-GRID METHOD FOR EIGENVALUE PROBLEMS
}

\author{
XIAOZHE HU AND XIAOLIANG CHENG
}

\begin{abstract}
This paper provides a new two-grid discretization method for solving partial differential equation or integral equation eigenvalue problems. In 2001, Xu and Zhou introduced a scheme that reduces the solution of an eigenvalue problem on a finite element grid to that of one single linear problem on the same grid together with a similar eigenvalue problem on a much coarser grid. By solving a slightly different linear problem on the fine grid, the new algorithm in this paper significantly improves the theoretical error estimate which allows a much coarser mesh to achieve the same asymptotic convergence rate. Numerical examples are also provided to demonstrate the efficiency of the new method.
\end{abstract}

\section{INTRODUCTION}

In this paper, we present an improved two-grid method for solving eigenvalue problems. The two-grid discretization method has been well developed in recent years. It was first introduced by $\mathrm{Xu}$ [17, 18, 19] for nonsymmetric and nonlinear elliptic problems. It was then applied to other problems by many researchers, including: Axelsson and Layton [1 for nonlinear elliptic problems, Dawson and Wheeler [7] for nonlinear parabolic equations, Layton and Lenferink [11, Utnes [16, and Layton and Tobiska [12] for Navier-Stokes problems, Marion and Xu [13] for evolution problems, and $\mathrm{Xu}$ and Zhou 22 for eigenvalue problems. This method is also used as part of the finite difference scheme (see also Dawson, Wheeler and Woodward [8] for parabolic equations). Recently, Chien and Jeng [6] used this method along with the continuation method for solving semilinear elliptic eigenvalue problems; Jin, Shu and Xu [10] employed it for decoupling systems of partial differential equations; Xu and Zhou [20, 21, 23] developed localized and parallelized algorithms based on two-grid discretizations for linear and nonlinear elliptic boundary problems as well as eigenvalue problems.

In this paper, we propose an improved two-grid discretization method for eigenvalue problems: Find $\lambda_{h} \in \mathbb{R}$ and $u_{h} \in S_{h} \backslash\{0\}$ satisfy

$$
a\left(u_{h}, v_{h}\right)=\lambda_{h} b\left(u_{h}, v_{h}\right), \quad \forall v_{h} \in S_{h} .
$$

Here $S_{h}$ is a finite element space defined on a quasi-uniform grid of size $h$. Expanding upon an idea developed by $\mathrm{Xu}$ and Zhou [22, we solve a standard finite element

Received by the editor October 21, 2009 and, in revised form, June 15, 2010.

2010 Mathematics Subject Classification. Primary 65L15, 65N15, 65N25, 65N30, 65N55.

Key words and phrases. Eigenvalue problems, finite elements, partial differential equations, integral equations, two-grid method.

This work was supported in part by National Science Foundation of China (No. 10871179).

(c)2011 American Mathematical Society

Reverts to public domain 28 years from publication 
discretization for an eigenvalue problem on a coarse space $S_{H}$ : Find $u_{H} \in S_{H}$, $\lambda_{H} \in \mathbb{R}$ such that

$$
a\left(u_{H}, v\right)=\lambda_{H} b\left(u_{H}, v\right), \quad \forall v \in S_{H},
$$

and obtain a rough approximation of the eigenpair. We then solve a linear problem based on $\left(\lambda_{H}, u_{H}\right)$ on a fine space $S_{h}$ : Find $u^{h} \in S_{h}$ such that

$$
a\left(u^{h}, v\right)-\lambda_{H} b\left(u^{h}, v\right)=b\left(u_{H}, v\right), \quad \forall v \in S_{h}, \text { and } \lambda^{h}=\frac{a\left(u^{h}, u^{h}\right)}{b\left(u^{h}, u^{h}\right)},
$$

and obtain a corrected eigenpair $\left(\lambda^{h}, u^{h}\right)$ instead of solving the eigenvalue problem on the fine grid directly. We obtain the following results (see Section 3) for elliptic eigenvalue problems:

$$
\min _{\alpha \in \mathbb{R}}\left\|u-\alpha u^{h}\right\|_{a}=O\left(h^{r}+H^{3 r+1}\right) \text { and }\left|\lambda^{h}-\lambda\right|=O\left(h^{2 r}+H^{6 r+2}\right),
$$

where $r$ is the degree of the piecewise polynomials. These estimates mean that we can obtain asymptotically optimal accuracy by using $H=O\left(h^{\frac{1}{4}}\right)$ if we use the piecewise linear finite element. In comparison with the results by Xu and Zhou 22]:

$$
\left\|u-u^{h}\right\|_{a}=O\left(h^{r}+H^{r+1}\right) \text { and }\left|\lambda^{h}-\lambda\right|=O\left(h^{2 r}+H^{2 r+2}\right),
$$

where the asymptotically optimal accuracy is obtained by taking $H=O\left(h^{\frac{1}{2}}\right)$ if the piecewise linear element is used. Our method can either use a coarser grid to obtain the same optimal accuracy or use the same coarse and fine grid to accelerate the convergence and obtain a better approximation for the eigenpair.

The rest of this paper is organized as follows. In Section 2, we describe the standard finite element method for eigenvalue problems. Section 3 contains the new algorithm of the paper, as well as error analysis and extensions. In Section 4 we give some numerical examples to show the efficiency of our new method.

\section{Preliminaries}

In this section, we describe some basic notation and properties of the standard finite element approximation of the self-adjoint eigenvalue problems.

2.1. Setting for the problem. Suppose that $\mathcal{H}$ is a real Hilbert space with an inner product $(\cdot, \cdot)$ and norm $\|\cdot\|$, respectively. Let $a(\cdot, \cdot)$ and $b(\cdot, \cdot)$ be two given symmetric bilinear forms on $\mathcal{H} \times \mathcal{H}$. Assume that $a(\cdot, \cdot)$ satisfies

$$
|a(u, v)| \leq C_{0}\|u\|\|v\|, \quad \forall u, v \in \mathcal{H},
$$

and there exists $C_{1}>0$ such that

$$
a(u, u) \geq C_{1}\|u\|^{2}, \quad \forall u \in \mathcal{H} .
$$

Then we can introduce another norm $\|u\|_{a}:=a(u, u)^{1 / 2}$ on $\mathcal{H}$. Obviously, $\|u\|_{a}$ and $\|u\|$ are two equivalent norms. In the rest of this paper, we will use $a(u, v)$ and $\|u\|_{a}$ as the inner product and norm on $\mathcal{H}$ and denote this space by $\mathcal{H}_{a}$. For $b(\cdot, \cdot)$, we assume that

$$
\|u\|_{b}:=b(u, u)^{1 / 2}
$$

defines a norm on $\mathcal{H}$. Let $\mathcal{H}_{b}$ be the completion of $\mathcal{H}_{a}$ with respect to $\|\cdot\|_{b}, \mathcal{H}_{b}$ is a Hilbert space with inner product $b(\cdot, \cdot)$ and $\|\cdot\|_{b}$ is assumed to be compact with respect to $\|\cdot\|_{a} \cdot \mathcal{H}_{a}$ is compactly embedded in $\mathcal{H}_{b}$. (Alternately, we can assume that $\mathcal{H}_{a} \subset \mathcal{H}_{b}$ is compact, and let $b(\cdot, \cdot)$ be the inner product on $\left.\mathcal{H}_{b}\right)$. 
From $\mathcal{H}_{a}$ and $\mathcal{H}_{b}$, we can construct the negative space $\mathcal{H}_{-a}=\mathcal{H}_{a}^{\prime}$ with a dual norm $\|\cdot\|_{-a}$ given by

$$
\|u\|_{-a}=\sup _{v \in \mathcal{H}_{a},\|v\|_{a}=1}|b(u, v)|,
$$

then $\mathcal{H}_{b} \subset \mathcal{H}_{-a}$ compactly, and for $v \in \mathcal{H}_{a}, b(u, v)$ has a continuous extension to $u \in \mathcal{H}_{-a}$ which results in the dual form $\langle\cdot, \cdot\rangle_{\mathcal{H}_{-a} \times \mathcal{H}_{a}}$ so that $b(u, v)$ is continuous on $\mathcal{H}_{-a} \times \mathcal{H}_{a}$. We can assign an inclusion, $I \in L\left(\mathcal{H}_{a}, \mathcal{H}_{-a}\right)$, to the bilinear form $b(u, v)$ as follows:

$$
b(u, v)=\langle I u, v\rangle_{\mathcal{H}_{-a} \times \mathcal{H}_{a}}=\langle u, v\rangle_{\mathcal{H}_{-a} \times \mathcal{H}_{a}}, \quad \forall u \in \mathcal{H}_{a}, v \in \mathcal{H}_{a} .
$$

By the negative space $\mathcal{H}_{-a}$ and (2.1), for the bilinear form $a(u, v)$, we can define a unique operator $A \in L\left(\mathcal{H}_{a}, \mathcal{H}_{-a}\right)$ such that

$$
a(u, v)=\langle A u, v\rangle_{\mathcal{H}_{-a} \times \mathcal{H}_{a}}, \quad \forall u, v \in \mathcal{H}_{a}, \quad \text { and }\|A\|_{\mathcal{H}_{a} \rightarrow \mathcal{H}_{-a}} \leq C_{0} .
$$

2.2. Finite element spaces. Assume that $\Gamma^{h}=\{\tau\}$ is a mesh of $\Omega$ and $h=$ $\max _{\tau \in \Gamma^{h}} h_{\tau}$ is the largest mesh size of $\Gamma^{h}$. Let the family $\left\{S_{h}\right\}$ be the finite element spaces on $\Omega$ associated with the mesh and satisfy the following approximation property: For any $u \in \mathcal{H}_{a}$, we get

$$
\lim _{h \rightarrow 0} \inf _{v \in S_{h}}\|u-v\|_{a}=0 .
$$

Let $P_{h}$ be the orthogonal projection of $\mathcal{H}_{a}$ onto $S_{h}$ with respect to the bilinear form $a(\cdot, \cdot)$, namely

$$
a(u, v)=a\left(P_{h} u, v\right), \quad \forall u \in \mathcal{H}_{a}, v \in S_{h}
$$

and, clearly,

$$
\left\|P_{h} u\right\|_{a} \leq\|u\|_{a}, \quad \forall u \in \mathcal{H}_{a} .
$$

If $u \in \mathcal{H}_{a}$, then

$$
\lim _{h \rightarrow 0}\left\|u-P_{h} u\right\|=0 .
$$

We also introduce two more operators, which are used in the analysis of our accelerated two-grid scheme, $T$ and $T_{h}: \mathcal{H}_{-a} \rightarrow \mathcal{H}_{a}$ such that

$$
a(T f, v)=b(f, v), \quad T f \in \mathcal{H}_{a}, \forall v \in \mathcal{H}_{a}
$$

and

$$
a\left(T_{h} f, v\right)=b(f, v), \quad T f \in S_{h}, \forall v \in S_{h} .
$$

Note that $T_{h}=P_{h} T$. Let $\eta(h)$ be defined by

$$
\eta(h)=\left\|T-T_{h}\right\|_{\mathcal{H}_{-a} \times \mathcal{H}_{a}}=\sup _{g \in \mathcal{H}_{a},\|g\|_{a}=1} \inf _{v \in S_{h}}\|T g-v\|_{a},
$$

which gives us the following result (see Lemma 3.3 and 3.4 in [2]):

Lemma 2.1. Let $\eta(h)$ be defined as in (2.10), then

$$
\lim _{h \rightarrow 0} \eta(h)=0
$$

and

$$
\left\|u-P_{h} u\right\|_{-a} \leq \eta(h)\left\|u-P_{h} u\right\|_{a}, \quad \forall u \in \mathcal{H}_{a} .
$$


2.3. Eigenvalue problems and some classical error estimates. We consider variationally formulated, self-adjoint eigenvalue problems. We call a real number $\lambda$ and a nonzero function $u$ an eigenpair of the bilinear form $a(\cdot, \cdot)$ with respect to the bilinear form $b(\cdot, \cdot)$ if they satisfy

$$
a(u, v)=\lambda b(u, v), \quad \forall v \in \mathcal{H}_{a} .
$$

Under the assumptions we have made, (2.11) has a countable sequence of real eigenvalues

$$
0<\lambda_{1} \leq \lambda_{2} \leq \cdots,
$$

and corresponding eigenvectors

$$
u_{1}, u_{2}, \cdots,
$$

which can be chosen to satisfy that

$$
a\left(u_{i}, u_{j}\right)=\lambda_{i} b\left(u_{i}, u_{j}\right)=\delta_{i j}, \quad i, j=1,2, \cdots .
$$

The eigenvalues and eigenvectors satisfy the following well-known variational principle known as the min-max principle:

$$
\lambda_{i}=\min _{V_{i} \in \mathcal{H}_{a}, \operatorname{dim}} \max _{i=i} \frac{a(u, u)}{b(u, u)} .
$$

Next we will give an approximation of the eigenpair of (2.11) using the standard finite element method, or more generally, the Galerkin method. To this end, suppose that we are given a family $\left\{S_{h}\right\}$ of finite-dimensional subspaces and $S_{h} \subset \mathcal{H}_{a}$. Consider the following eigenvalue problem: Find $\lambda_{h} \in \mathbb{R}$ and $u_{h} \in S_{h} \backslash\{0\}$ satisfy

$$
a\left(u_{h}, v_{h}\right)=\lambda_{h} b\left(u_{h}, v_{h}\right), \quad \forall v_{h} \in S_{h} .
$$

The eigenpair $\left(\lambda_{h}, u_{h}\right)$ of (2.13) will be viewed as an approximation of the eigenpair $(\lambda, u)$ of (2.11) (as $h \rightarrow 0)$. (2.13) has a sequence of eigenvalues

$$
0<\lambda_{h, 1} \leq \lambda_{h, 2} \leq \cdots \lambda_{h, N_{h}}, \quad N_{h}=\operatorname{dim} S_{h},
$$

and corresponding eigenvectors

$$
u_{h, 1}, u_{h, 2}, \cdots, u_{h, N_{h}}
$$

which can be chosen to satisfy

$$
a\left(u_{h, i}, u_{h, j}\right)=\lambda_{h, i} b\left(u_{h, i}, u_{h, j}\right)=\delta_{i j}, \quad i, j=1,2, \cdots, N_{h} .
$$

The min-max principle analogous to (2.12) holds for (2.13) by replacing $\mathcal{H}_{a}$ by $S_{h}$ and taking $i=1,2, \cdots, N_{h}$. It follows directly from the min-max principle that

$$
\lambda_{i} \leq \lambda_{h, i}, \quad i=1,2, \cdots, N_{h} .
$$

It follows from (2.6) that

$$
\lambda_{h, i} \rightarrow \lambda_{i}, \quad \text { as } h \rightarrow 0
$$

Assuming $\lambda_{i}$ has multiplicity $q_{i}$, let

$$
M\left(\lambda_{i}\right)=\left\{u \in \mathcal{H}_{a}: u \text { is an eigenvector of (2.11) corresponding to } \lambda_{i}\right\},
$$

and

$$
\delta_{h}\left(\lambda_{i}\right)=\sup _{u \in M\left(\lambda_{i}\right),\|u\|_{a}=1} \inf _{u \in S_{h}}\|u-v\|_{a} .
$$

$\lambda_{i}$ is approximated by $\lambda_{h, i+j}, j=0, \cdots, q_{i}-1$. Let $u_{h, i+j}$ denote the approximate eigenvectors corresponding to $\lambda_{h, i+j}, j=0, \cdots, q_{i}-1$. Similarly, let

$$
M_{h}\left(\lambda_{i}\right)=\operatorname{span}\left\{u_{h, i}, u_{h, i+1}, \cdots, u_{h, i+q_{i}-1}\right\},
$$


and $E_{h}$ be the orthogonal projection of $\mathcal{H}_{a}$ onto $M_{h}\left(\lambda_{i}\right)$.

The following result is well known (see p. 699 of [3] and Lemma 3.6, 3.7 and (3.29b) of [2], or cf. [4]) and is useful in error analysis.

Lemma 2.2. (i) For any $u_{h, i} \in M_{h}\left(\lambda_{i}\right)$ of (2.13) $\left(i=1,2, \cdots, N_{h}\right)$, there is an eigenvector $u_{i} \in M\left(\lambda_{i}\right)$ of (2.11) corresponding to $\lambda_{i}$ and

$$
\left\|u_{i}-u_{h, i}\right\|_{a} \leq C_{i} \delta_{h}\left(\lambda_{i}\right)
$$

or for any $u_{i} \in M\left(\lambda_{i}\right)$,

$$
\left\|u_{i}-E_{h} u_{i}\right\|_{a} \leq C_{i} \delta_{h}\left(\lambda_{i}\right) .
$$

Moreover,

$$
\left\|u_{i}-u_{h, i}\right\|_{-a} \leq C_{i} \eta(h)\left\|u_{i}-u_{h, i}\right\|_{a} .
$$

(ii) For the eigenvalue, we have

$$
\lambda_{i} \leq \lambda_{h, i} \leq \lambda_{i}+C_{i} \delta_{h}^{2}\left(\lambda_{i}\right) .
$$

Here and after $C_{i}$ is a constant which is dependent on $i$, but not dependent on $h$.

At the end of this section, we give an important but straightforward identity that relates the errors in the eigenvalue and eigenvector approximation (for examples, see Lemma 3.1 of [2] or Lemma 9.1 of [3]).

Proposition 2.3. Let $(\lambda, u)$ be an eigenpair of (2.11). For any $w \in \mathcal{H}_{a} \backslash\{0\}$, we have

$$
\frac{a(w, w)}{b(w, w)}-\lambda=\frac{a(w-u, w-u)}{b(w, w)}-\lambda \frac{b(w-u, w-u)}{b(w, w)} .
$$

\section{AN IMPROVED TWO-GRID DISCRETIZATION}

In this section, we present an accelerated scheme of two-grid discretization for eigenvalue problems. As in other two-grid methods, the basic mechanisms in our approach consist of two quasi-uniform triangulations of $\Omega, \Gamma_{H}$ and $\Gamma_{h}$ with different mesh sizes $H$ and $h(H>h)$ and their corresponding finite element spaces $S_{H}$ and $S_{h}$, also known as the coarse and fine spaces, respectively. We assume that $S_{H}$ and $S_{h}$ satisfy (2.6) and $S_{H} \subset S_{h}$.

3.1. An accelerated two-grid scheme and error estimate. We define a new bilinear form as follows:

$$
a_{\mu}(\cdot, \cdot): \mathcal{H}_{-a} \times \mathcal{H}_{a} \rightarrow \mathbb{R}, \quad a_{\mu}(u, v)=a(u, v)-\mu b(u, v) .
$$

Since $\mathcal{H}_{a} \subset \mathcal{H}_{b} \subset \mathcal{H}_{-a}$ compactly, it follows from (2.5) and (2.4) that

$$
a_{\mu}(u, v)=\langle(A-\mu I) u, v\rangle_{\mathcal{H}_{-a} \times \mathcal{H}_{a}}, \quad \forall u \in \mathcal{H}_{a}, \quad v \in \mathcal{H}_{a},
$$

and we have the following lemma for the newly introduced bilinear form:

Lemma 3.1. If $\mu$ is not an eigenvalue, there exist two constants $C(\mu)$ and $C_{h}(\mu)$ such that

$$
\sup _{v \in \mathcal{H}_{a}} \frac{\left|a_{\mu}(u, v)\right|}{\|v\|_{a}} \geq C(\mu)\|u\|_{a}, \quad \forall u \in \mathcal{H}_{a}
$$

and

$$
\sup _{v \in S_{h}} \frac{\left|a_{\mu}(u, v)\right|}{\|v\|_{a}} \geq C_{h}(\mu)\|u\|_{a}, \quad \forall u \in S_{h} .
$$


Proof. By (2.2), the operator defined by (2.5) satisfies that $A \in L\left(\mathcal{H}_{a}, \mathcal{H}_{-a}\right)$ and $A^{-1} \in L\left(\mathcal{H}_{-a}, \mathcal{H}_{a}\right)$. Since $\mathcal{H}_{a}$ is compactly embedded in $\mathcal{H}_{b}$, and $\mathcal{H}_{b}$ is compactly embedded in $\mathcal{H}_{-a}, \mathcal{H}_{a} \subset \mathcal{H}_{-a}$ is also a compact embedding (i.e, the inclusion $I \in L\left(\mathcal{H}_{a}, \mathcal{H}_{-a}\right)$ is compact). Hence, the operator $T$ defined by (2.8) satisfies $T=A^{-1} I: \mathcal{H}_{a} \rightarrow \mathcal{H}_{a}$ and is also compact from $\mathcal{H}_{a}$ to $\mathcal{H}_{a}$. Therefore, the RieszSchauder theory is applicable to $T-\nu I$. Since

$$
T-\nu I=-\nu\left(I-\nu^{-1} T\right)=-\nu A^{-1}(A-\mu I)
$$

with $\mu=1 / \nu$, the statements of the Riesz-Schauder theory transfer via $T-\nu I$ to $A-\mu I=-\nu^{-1} A(T-\nu I) .(A-\mu I)^{-1} \in L\left(\mathcal{H}_{-a}, \mathcal{H}_{a}\right)$, which means that (3.1) holds. For (3.2), note that the operator $T_{h}$ is also compact from $\mathcal{H}_{a}$ to $\mathcal{H}_{a}$, and hence the conclusion is obtained directly by replacing $A$ by $A_{h}$.

According to (3.1) and (3.2), if $\mu$ is not an eigenvalue, then $a_{\mu}(u, v)=\langle f, v\rangle$ is uniquely solvable for all $v \in \mathcal{H}_{a}$ or $v \in S_{h}$. If $\mu$ is an eigenvalue, then $a_{\mu}(u, v)=$ $\langle f, v\rangle$ may have no solution (In fact, it has at least one solution if and only if $\left.f \in M(\mu)^{\perp}\right)$.

Now, the main algorithm of this paper is presented as follows:

Algorithm 1. Accelerated Two-grid Method

Step 1: Find $\lambda_{i, H} \in \mathbb{R}$ and $u_{i, H} \in S_{H}\left(i=1,2, \cdots, N_{H}\right)$ such that $\left\|u_{i, H}\right\|_{a}=1$ and

$$
a\left(u_{i, H}, v\right)=\lambda_{i, H} b\left(u_{i, H}, v\right), \quad \forall v \in S_{H} .
$$

Step 2: Find $u_{i}^{h} \in S_{h},\left(i=1,2, \cdots, N_{H}\right)$, such that

$$
a_{\lambda_{i, H}}\left(u_{i}^{h}, v\right)=b\left(u_{i, H}, v\right), \quad \forall v \in S_{h} .
$$

Step 3: Set

$$
\lambda_{i}^{h}=\frac{a\left(u_{i}^{h}, u_{i}^{h}\right)}{b\left(u_{i}^{h}, u_{i}^{h}\right)}, \quad i=1,2, \cdots, N_{H}
$$

Remark 3.2. We note that the linear system in Step 2 is nearly singular and how this system is solved efficiently is obviously a matter of concern. This problem has been much discussed in the literature in the context of the general inverse power method. As shown in, e.g., 9, 15, 14, the near-singularity of this system seldomly presents a problem for the inverse power method. Let $x_{1}$ and $\tilde{x}_{1}$ denote the exact and computed solution of one step of the inverse power method with shift $\sigma$. Because of the roundoff error, we have $(A-\sigma I) x_{1}=x_{0}$, and $(A-\sigma I) \tilde{x}_{1}=x_{0}+g$. Let $\phi$ be the angle between $g$ and the eigenvector $v_{i}$, and the orthogonal decomposition of $g$ is $g=\left(v_{i} \cos \phi+z \sin \phi\right)\|g\|$, where $z^{T} v_{i}=0$. Therefore, we have a similar orthogonal decomposition for the error $e=\tilde{x}_{1}-x_{1}=(A-\sigma I)^{-1} g$ :

$$
e=\left(v_{i} \cos \phi /\left(\lambda_{i}-\sigma\right)+\tilde{z}\left\|(A-\sigma I)^{-1} z\right\| \sin \phi\right)\|g\|,
$$

where $\tilde{z}$ is the normalized version of $(A-\sigma I)^{-1} z$ and is orthogonal to $v_{i}$. Let $\psi$ denote the angle between $e$ and $v_{i}$, then we have

$$
\tan \psi=\left\|(A-\sigma I)^{-1} z\right\|\left(\lambda_{i}-\sigma\right) \tan \phi \leq \frac{\left|\lambda_{i}-\sigma\right|}{\min _{j \neq i}\left|\lambda_{j}-\sigma\right|} \tan \phi .
$$

This shows that the error $e$ is almost entirely in the direction of the eigenvector $v_{i}$ when $\left|\lambda_{i}-\sigma\right|$ is very small. Therefore, an iterative method (such as the multigrid 
method or the conjugate gradient method) can be used to solve this nearly singular system without much extra difficulty. Moreover, when this system actually becomes singular or very close to being singular, $\lambda_{i, H}$ will be equal or very close to $\lambda_{i, h}$, it means that $\lambda_{i, H}$ is already a good approximation of $\lambda_{i, h}$.

The following theorem gives the error estimates for our accelerated two-grid scheme.

Theorem 3.3. Assume that $\left(\lambda_{i}^{h}, u_{i}^{h}\right),\left(i=1,2, \cdots, N_{H}\right)$ are calculated via Algorithm 1. If $S_{H} \subset S_{h}$, then there exists an eigenvector $u_{i} \in M\left(\lambda_{i}\right)$ such that

$$
\begin{aligned}
\min _{\alpha \in \mathbb{R}}\left\|u_{i}-\alpha u_{i}^{h}\right\|_{a} & \leq C_{i}\left(\left\|u_{i}-P_{h} u_{i}\right\|_{-a}+\left|\lambda_{i, H}-\lambda_{i}\right|\left\|u_{i}-P_{h} u_{i}\right\|_{-a}\right. \\
& \left.+\left|\lambda_{i, H}-\lambda_{i}\right|\left\|u_{i}-u_{i, H}\right\|_{-a}+\left\|u_{i}-P_{h} u_{i}\right\|_{a}\right) .
\end{aligned}
$$

For the eigenvalue, we have

$$
\begin{aligned}
\left|\lambda_{i}^{h}-\lambda_{i}\right| & \leq C_{i}\left(\left\|u_{i}-P_{h} u_{i}\right\|_{-a}+\left|\lambda_{i, H}-\lambda_{i}\right|\left\|u_{i}-P_{h} u_{i}\right\|_{-a}\right. \\
& \left.+\left|\lambda_{i, H}-\lambda_{i}\right|\left\|u_{i}-u_{i, H}\right\|_{-a}+\left\|u_{i}-P_{h} u_{i}\right\|_{a}\right)^{2} .
\end{aligned}
$$

Consequently,

$$
\begin{gathered}
\min _{\alpha \in \mathbb{R}}\left\|u_{i}-\alpha u_{i}^{h}\right\|_{a} \leq C_{i}\left(\eta(h) \delta_{h}\left(\lambda_{i}\right)+\delta_{H}^{2}\left(\lambda_{i}\right) \delta_{h}\left(\lambda_{i}\right) \eta(h)+\delta_{H}^{3}\left(\lambda_{i}\right) \eta(H)+\delta_{h}\left(\lambda_{i}\right)\right), \\
3.7) \quad\left|\lambda_{i}^{h}-\lambda_{i}\right| \leq C_{i}\left(\eta^{2}(h) \delta_{h}^{2}\left(\lambda_{i}\right)+\delta_{H}^{4}\left(\lambda_{i}\right) \delta_{h}^{2}\left(\lambda_{i}\right) \eta^{2}(h)+\delta_{H}^{6}\left(\lambda_{i}\right) \eta^{2}(H)+\delta_{h}^{2}\left(\lambda_{i}\right)\right) .
\end{gathered}
$$

Proof. Consider an equivalent linear system on the fine grid as follows:

$$
a_{\lambda_{i, H}}\left(\widetilde{u}_{i}^{h}, v\right)=\left(\lambda_{i}-\lambda_{i, H}\right) b\left(u_{i, H}, v\right), \quad \forall v \in S_{h} .
$$

Note that

$$
\lambda_{i}^{h}=\frac{a\left(u_{i}^{h}, u_{i}^{h}\right)}{b\left(u_{i}^{h}, u_{i}^{h}\right)}=\frac{a\left(\widetilde{u}_{i}^{h}, \widetilde{u}_{i}^{h}\right)}{b\left(\widetilde{u}_{i}^{h}, \widetilde{u}_{i}^{h}\right)} .
$$

According to Lemma 2.2 there exists an eigenvector $u_{i} \in M\left(\lambda_{i}\right)$ such that $\| u_{i}-$ $u_{i, H} \| \leq C_{i} \delta_{H}\left(\lambda_{i}\right)$. From (2.11), (2.7) and (3.8), we have

$$
\begin{aligned}
a_{\lambda_{i, H}}\left(P_{h} u_{i}-\widetilde{u}_{i}^{h}, v\right) & =\lambda_{i} b\left(u_{i}-P_{h} u_{i}, v\right)+\left(\lambda_{i, H}-\lambda_{i}\right) b\left(u_{i}-P_{h} u_{i}, v\right) \\
& +\left(\lambda_{i}-\lambda_{i, H}\right) b\left(u_{i}-u_{i, H}, v\right), \quad \forall v \in S_{h} .
\end{aligned}
$$

From Lemma 3.1 and (2.3), we can obtain

$$
\begin{aligned}
\left\|u_{i}-\widetilde{u}_{i}^{h}\right\|_{a} \leq & C_{i}\left(\left\|u_{i}-P_{h} u_{i}\right\|_{-a}+\left|\lambda_{i, H}-\lambda_{i}\right|\left\|u_{i}-P_{h} u_{i}\right\|_{-a}\right. \\
& \left.+\left|\lambda_{i, H}-\lambda_{i}\right|\left\|u_{i}-u_{i, H}\right\|_{-a}+\left\|u_{i}-P_{h} u_{i}\right\|_{a}\right) .
\end{aligned}
$$

Note that $\min _{\alpha \in \mathbb{R}}\left\|u_{i}-\alpha u_{i}^{h}\right\|_{a} \leq\left\|u_{i}-\widetilde{u}_{i}^{h}\right\|_{a}$, 3.4 follows from (3.11) immediately. (3.5) follows from (3.9), (3.11), and Proposition 2.3. Finally, (3.6) and (3.7) are obtained by Lemma 2.2

Remark 3.4. Our accelerated two-grid scheme expands upon the idea developed by $\mathrm{Xu}$ and Zhou 22. Therefore, we can also extend it to nonselfadjoint eigenvalue problems. Assume that $a(\cdot, \cdot)$ may not be symmetric, but $b(\cdot, \cdot)$ is symmetric. Note that

$$
\frac{a(w, w)}{b(w, w)}-\lambda=\frac{a(w-u, w-u)}{b(w, w)}-\lambda \frac{b(w-u, w-u)}{b(w, w)}+\frac{a(w-u, u)-a(u, w-u)}{b(w, w)} .
$$


For instance, when $r>1$ and the bilinear form $a(\cdot, \cdot)$ corresponds to a general elliptic operator of second order, then

$$
|a(w-u, u)-a(u, w-u)| \leq C\|w-u\|_{1-r}
$$

and

$$
\|w-u\|_{1-r} \ll\|w-u\|_{1} .
$$

Therefore, in this situation, our accelerated two-grid scheme can also be applied to nonselfadjoint eigenvalue problems.

3.2. Examples. Our accelerated two-grid method is suitable for a large class of self-adjoint eigenvalue problems. Next, we give two examples: One is a partial differential operator and the other is an integral operator. Let $\Omega \subset \mathbb{R}^{d}(d=1,2, \cdots)$ be a bounded polygonal and convex domain, and $\Gamma^{h}(\Omega)$, consisting of shape-regular simplices, be a mesh with mesh size $h$.

\subsubsection{Second order elliptic operators. Define}

$$
\begin{aligned}
a(u, v) & =\int_{\Omega} \sum_{i, j=1}^{d} a_{i j} \frac{\partial u}{\partial x_{i}} \frac{\partial v}{\partial x_{j}}, \quad a_{i j} \in W_{1, \infty}(\Omega), \\
b(u, v) & =(u, v)_{L^{2}},
\end{aligned}
$$

where $A=\left(a_{i j}\right)$ is uniformly positive definite on $\Omega$. Introduce the following spaces

$$
\mathcal{H}_{a}=H_{0}^{1}(\Omega), \quad \mathcal{H}_{b}=L^{2}(\Omega),
$$

and the finite element space

$$
S_{h}(\Omega)=\left\{v \in C(\bar{\Omega}) \cap H_{0}^{1}(\Omega):\left.v\right|_{\tau} \in P_{\tau}^{r}, \quad \forall \tau \in \Gamma_{h}(\Omega)\right\},
$$

where $P_{\tau}^{r}$ is the space of polynomials whose degree is not greater than the positive integer $r$.

Assume $M\left(\lambda_{i}\right) \subset H^{r+1}(\Omega)$, and then

$$
\eta(h)=O(h), \quad \delta_{h}\left(\lambda_{i}\right) \leq C_{i} h^{r} .
$$

Hence,

$$
\min _{\alpha \in \mathbb{R}}\left\|u_{i}-\alpha u_{i}^{h}\right\|_{a} \leq C_{i}\left(h^{r}+H^{3 r+1}\right)
$$

and

$$
\left|\lambda_{i}^{h}-\lambda_{i}\right| \leq C_{i}\left(h^{2 r}+H^{6 r+2}\right) .
$$

If we use the piecewise linear finite element, namely $r=1$, then the asymptotically optimal accuracy is obtained by taking $H=h^{\frac{1}{4}}$.

Remark 3.5. In [22, the error estimates of the eigenvector and eigenvalue are as follows:

$$
\left\|u_{i}-u_{i}^{h}\right\|_{a} \leq C_{i}\left(h^{r}+H^{r+1}\right)
$$

and

$$
\left|\lambda_{i}^{h}-\lambda_{i}\right| \leq C_{i}\left(h^{2 r}+H^{2 r+2}\right) .
$$

This means that the asymptotically optimal accuracy is obtained by taking $H=$ $h^{\frac{r}{r+1}}$ (when $r=1, H=h^{\frac{1}{2}}$ ). When $r$ is large (such as in the case of a high order finite element), we have $h \approx H$, which means we cannot use a coarse grid; otherwise improvement stemming from correction on the fine grid will be inconspicuous. 
But with our accelerated two-grid scheme, the asymptotically optimal accuracy is obtained by taking $H=h^{\frac{r}{3 r+1}}$. Obviously, our scheme accelerates the convergence. Even when $r$ is large, we still have $h \approx H^{3}$, and hence the improvement on the fine grid will still be conspicuous.

3.2.2. Fredholm integral operators. Define

$$
\begin{aligned}
a(u, v) & =(u, v)_{L^{2}}, \\
b(u, v) & =(k u, v)_{L^{2}},
\end{aligned}
$$

where $(k u)(x)=\int_{\Omega} k(x, y) u(x) \mathrm{d} y$ is the symmetric and positive definite Fredholm integral operator on $L^{2}(\Omega)\left(k(x, y) \in C^{r+1}(\Omega \times \Omega)\right)$. We introduce the spaces as follows:

$$
\mathcal{H}_{a}=L^{2}(\Omega), \quad \mathcal{H}_{b}=\text { the completion of } \mathcal{H}_{a} \text { with respect to }\|\cdot\|_{b}
$$

and

$$
S_{h}(\Omega)=\left\{v \in C(\bar{\Omega}) \cap H_{0}^{1}(\Omega):\left.v\right|_{\tau} \in P_{\tau}^{r}, \quad \forall \tau \in \Gamma_{h}(\Omega)\right\} .
$$

Then we have

$$
\eta(h)=O\left(h^{r+1}\right), \quad \delta_{h}\left(\lambda_{i}\right) \leq C_{i} h^{r+1}
$$

and, therefore,

$$
\min _{\alpha \in \mathbb{R}}\left\|u_{i}-\alpha u_{i}^{h}\right\|_{a} \leq C_{i}\left(h^{r+1}+H^{4 r+4}\right)
$$

and

$$
\left|\lambda_{i}^{h}-\lambda_{i}\right| \leq C_{i}\left(h^{2 r+2}+H^{8 r+8}\right) .
$$

Remark 3.6. The error estimates obtained by Xu and Zhou in [22] for the Fredholm integral operator are

$$
\left\|u_{i}-u_{i}^{h}\right\|_{a} \leq C_{i}\left(h^{r+1}+H^{2 r+2}\right)
$$

and

$$
\left|\lambda_{i}^{h}-\lambda_{i}\right| \leq C_{i}\left(h^{2 r+2}+H^{4 r+4}\right) .
$$

Thus, it is proven that our method accelerates the convegence.

\section{NumERICAL EXAMPLES}

In this section, we present some numerical experiments for second order elliptic operators and demonstrate the efficiency of our accelerated two-grid algorithm. All of the numerical tests are based on AFEM@matlab [5].

\subsection{Example 1.}

$$
\begin{aligned}
-\Delta u & =\lambda u, \quad \text { in } \Omega, \\
u & =0, \quad \text { on } \partial \Omega,
\end{aligned}
$$

where $\Omega \subset \mathbb{R}$ or $\subset \mathbb{R}^{2}$.

(i) $\boldsymbol{\Omega}=(\mathbf{0}, \mathbf{1}) \times(\mathbf{0}, \mathbf{1})$. We consider the two-dimensional case $\Omega=(0,1) \times(0,1)$, where the eigenvalues are

$$
\lambda_{k, l}=\left(k^{2}+l^{2}\right) \pi^{2}, \quad k, l=1,2, \cdots .
$$

The corresponding eigenvectors are

$$
u_{k}=\sin k \pi x \sin l \pi y, \quad k, l=1,2, \cdots .
$$

We use the piecewise linear finite element space in the following numerical experiments. 
We first show the convergence rate of our accelerated two-grid scheme. According to Theorem 3.3, we have

$$
\min _{\alpha \in \mathbb{R}}\left\|\nabla\left(u_{h}-\alpha u^{h}\right)\right\|_{L^{2}}=O\left(H^{4}\right) \text { and }\left|\lambda^{h}-\lambda_{h}\right|=O\left(H^{8}\right),
$$

where $\left(\lambda_{h}, u_{h}\right)$ is the eigenpair obtained by solving the eigenvalue problem on the fine grid directly. The results shown in Table 1 consist of these estimates.

TABLE 1. Convergence rate test on uniform grid

\begin{tabular}{ccccc}
\hline \hline $\mathrm{H}$ & $\min _{\alpha}\left\|\nabla\left(u_{h}-\alpha u_{h}\right)\right\|_{L^{2}}$ & Ratio & $\left|\lambda^{h}-\lambda_{h}\right|$ & Ratio \\
\hline $1 / 2$ & $3.5948 \mathrm{E}-1$ & - & $1.0528 \mathrm{E}-1$ & - \\
$1 / 4$ & $2.4495 \mathrm{E}-2$ & $2^{3.8753}$ & $4.6979 \mathrm{E}-4$ & $2^{7.8080}$ \\
$1 / 8$ & $1.7281 \mathrm{E}-3$ & $2^{3.8253}$ & $2.2809 \mathrm{E}-6$ & $2^{7.6862}$ \\
$1 / 16$ & $1.1206 \mathrm{E}-4$ & $2^{3.9468}$ & $9.5479 \mathrm{E}-9$ & $2^{7.9002}$ \\
\hline \hline
\end{tabular}

In order to demonstrate the acceleration of our new proposed scheme and compare our numerical results fairly with those of $\mathrm{Xu}$ and Zhou, we first apply both schemes on the same uniform coarse and fine grid satisfying $H^{2}=h$. (By taking $H^{2}=h, \mathrm{Xu}$ and Zhou's scheme can obtain asymptotically optimal accuracy.) In addition, we apply our accelerated scheme on coarser grids $H=\frac{1}{4}, \frac{1}{8}$ with the fine grids $h=\frac{1}{64}, \frac{1}{256}$ to show that we can obtain better results by applying our accelerated scheme using a coarser grid and the same fine grid. Also, in order to show that our accelerated scheme can improve the results on a large class of coarse and fine grids, we choose mesh sizes satisfying $h=H / 2$, a common occurrence in the mesh refinement process. Finally, we apply two schemes on the unstructured meshes (see Figure 11), and the results are shown in Table 2. Here, $\lambda$ denotes the exact eigenvalue, and $\lambda_{h}$ is the standard finite element eigenvalue on the fine grid. $\lambda_{X Z}$ and $\lambda_{A c}$ denote the approximate eigenvalues obtained by $\mathrm{Xu}$ and Zhou's scheme and our accelerated scheme, respectively.

From Table 2, we can see that our accelerated two-grid scheme outperforms in all cases. Although our accelerated scheme cannot obtain asymptotically optimal accuracy when $H=h^{\frac{1}{2}}$, we can still get a better approximate eigenvalue. Furthermore, we can use coarser grids $(H=1 / 4, h=1 / 64$ and $H=1 / 8, h=256)$ to obtain better approximations. For grids obtained by the mesh refinement procedure $(H=2 h)$ or unstructured grids, our accelerated scheme still works better.

Table 3 shows the error of the eigenvector approximation. We can see that in all of cases, our accelerated scheme provides a better approximate eigenvector. Table 3 also lists the CPU time for the two schemes. Here we only show the computational time on the fine grid and the direct solver (")" in MATLAB) is used. On the same coarse and fine grids, our accelerated scheme may take a little bit more time. However, the eigenpair approximation obtained by our new scheme is much better. Moreover, our accelerated scheme can use coarser grids $(H=1 / 8, h=1 / 16$ for our new scheme and $H=1 / 32, h=1 / 64$ for $\mathrm{Xu}$ and Zhou's scheme) to produce similar results $\left(\lambda_{A c}-\lambda_{h}=7.22 \mathrm{E}-7, \lambda_{X Z}-\lambda_{h}=2.14 \mathrm{E}-6, \min _{\alpha}\left\|\nabla\left(u_{h}-\alpha u_{A c}^{h}\right)\right\|_{L^{2}}=\right.$ $9.67 \mathrm{E}-4$ and $\left.\min _{\alpha}\left\|\nabla\left(u_{h}-\alpha u_{X z}^{h}\right)\right\|_{L^{2}}=1.67 \mathrm{E}-3\right)$, which saves the computational time (1.00E-2 seconds for our accelerated scheme and 1.6E-1 seconds for Xu and Zhou's scheme). 


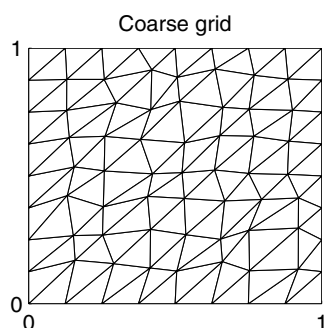

(a)

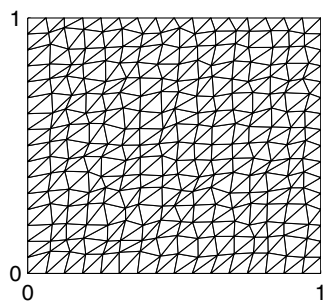

(c)

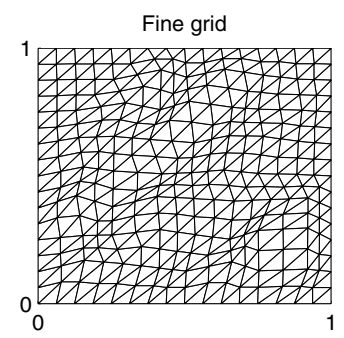

(b)

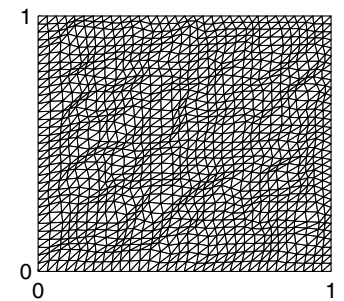

(d)

Figure 1. Unstructured coarse and fine meshes. (a) and (b) are a pair of coarse and fine grids, (c) and (d) constitute another pair.

TABLE 2. Results of Example 1 on $\Omega=(0,1) \times(0,1)$ for the first eigenvalue $\lambda=19.739208802178716$

\begin{tabular}{|c|c|c|c|c|c|}
\hline Coarse & Fine & $\lambda_{X Z}$ & $\lambda_{A c}$ & $\lambda_{X Z}-\lambda_{h}$ & $\lambda_{A c}-\lambda_{h}$ \\
\hline $\mathrm{H}$ & $\mathrm{h}$ & \multicolumn{4}{|c|}{ Coarse and fine grids satisfying $H=h^{\frac{1}{2}}$} \\
\hline $1 / 4$ & $1 / 16$ & 19.942337 & 19.930260 & $1.25 \mathrm{E}-2$ & $4.70 \mathrm{E}-4$ \\
\hline $1 / 8$ & $1 / 64$ & 19.752004 & 19.751103 & $9.03 \mathrm{E}-4$ & $2.28 \mathrm{E}-7$ \\
\hline $1 / 16$ & $1 / 256$ & 19.740012 & 19.739952 & $6.00 \mathrm{E}-5$ & $9.55 \mathrm{E}-9$ \\
\hline $\mathrm{H}$ & $\mathrm{h}$ & \multicolumn{4}{|c|}{ Coarser grid (only for our accelerated scheme) $H>h^{\frac{1}{2}}$} \\
\hline $1 / 4$ & $1 / 64$ & - & 19.751738 & - & $6.37 \mathrm{E}-4$ \\
\hline $1 / 8$ & $1 / 256$ & - & 19.739954 & - & $2.43 \mathrm{E}-6$ \\
\hline $\mathrm{H}$ & $\mathrm{h}$ & \multicolumn{4}{|c|}{ Coarse and fine grids satisfying $H=2 h$} \\
\hline $1 / 4$ & $1 / 8$ & 20.513575 & 20.505713 & $8.03 \mathrm{E}-3$ & $1.68 \mathrm{E}-4$ \\
\hline $1 / 8$ & $1 / 16$ & 19.930303 & 19.929791 & $5.13 \mathrm{E}-4$ & $7.22 \mathrm{E}-7$ \\
\hline $1 / 16$ & $1 / 32$ & 19.786826 & 19.786792 & $3.37 \mathrm{E}-5$ & $3.02 \mathrm{E}-9$ \\
\hline $1 / 32$ & $1 / 64$ & 19.751103 & 19.751101 & $2.14 \mathrm{E}-6$ & $1.18 \mathrm{E}-11$ \\
\hline $1 / 64$ & $1 / 128$ & 19.74218171 & 19.74218157 & $1.35 \mathrm{E}-7$ & $-7.35 \mathrm{E}-13$ \\
\hline $1 / 128$ & $1 / 256$ & 19.73995199 & 19.73995197 & $8.42 \mathrm{E}-9$ & $-3.37 \mathrm{E}-12$ \\
\hline Fig[1] & Fig[1] & \multicolumn{4}{|c|}{ Unstructured coarse and fine grids (see Figure 1) } \\
\hline (a) & (b) & 19.969542 & 19.968661 & $8.83 \mathrm{E}-4$ & $1.80 \mathrm{E}-6$ \\
\hline (c) & (d) & 19.793019 & 19.792981 & $3.79 \mathrm{E}-5$ & $4.36 \mathrm{E}-9$ \\
\hline
\end{tabular}

(ii) $\mathbf{\Omega}=(-\mathbf{1}, \mathbf{1}) \times(-\mathbf{1}, \mathbf{1}) \backslash(\mathbf{0}, \mathbf{1}) \times(-\mathbf{1}, \mathbf{0})$. We consider eigenvalue problems on an L-shaped domain in this section. The coarse grid used in this numerical experiment is chosen to be a uniform grid (see Figure 2(a)). Note that the eigenvector 
TABLE 3. Results of Example 1 on $\Omega=(0,1) \times(0,1)$ for the eigenvector corresponding to the first eigenvalue and CPU time (s)

\begin{tabular}{|c|c|c|c|c|c|}
\hline Coarse & Fine & $\overline{\min _{\alpha}\left\|\nabla\left(u_{h}-\alpha u_{X Z}^{h}\right)\right\|_{L^{2}} \min _{\alpha}}$ & $\left\|\nabla\left(u_{h}-\alpha u_{A c}^{h}\right)\right\|_{L^{2}}$ & 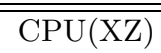 & $\mathrm{CPU}(\mathrm{Ac})$ \\
\hline $\mathrm{H}$ & $\mathrm{h}$ & \multicolumn{4}{|c|}{ Coarse and fine grids satisfying $H=h^{\frac{1}{2}}$} \\
\hline $1 / 4$ & $1 / 16$ & $1.25 \mathrm{E}-1$ & $2.45 \mathrm{E}-2$ & $2.00 \mathrm{E}-2$ & $2.00 \mathrm{E}-2$ \\
\hline $1 / 8$ & $1 / 64$ & E-2 & 1.731 & $1.30 \mathrm{E}-1$ & $2.00 \mathrm{E}-1$ \\
\hline $1 / 16$ & $1 / 256$ & $8.85 \mathrm{E}-3$ & $1.12 \mathrm{E}-4$ & $2.02 \mathrm{E} 0$ & $3.60 \mathrm{E} 0$ \\
\hline $\mathrm{H}$ & $\mathrm{h}$ & \multicolumn{4}{|c|}{ Coarse and fine grids satisfying $H=2 h$} \\
\hline $1 / 4$ & $1 / 8$ & $9.88 \mathrm{E}-2$ & $1.44 \mathrm{E}-2$ & $0.00 \mathrm{E} 0$ & $0.00 \mathrm{E} 0$ \\
\hline $1 / 8$ & $1 / 16$ & $2.57 \mathrm{E}-2$ & 9.67 & $9.99 \mathrm{E}-3$ & $1.00 \mathrm{E}-2$ \\
\hline $1 / 16$ & $1 / 32$ & $6.63 \mathrm{E}-3$ & $6.29 \mathrm{E}-5$ & $4.00 \mathrm{E}-2$ & $5.00 \mathrm{E}-2$ \\
\hline $1 / 32$ & $1 / 64$ & $1.67 \mathrm{E}-3$ & $3.98 \mathrm{E}-6$ & $1.60 \mathrm{E}-1$ & $1.90 \mathrm{E}-1$ \\
\hline $1 / 64$ & $1 / 128$ & $4.20 \mathrm{E}-4$ & $2.49 \mathrm{E}-7$ & $5.40 \mathrm{E}-1$ & $8.30 \mathrm{E}-1$ \\
\hline $1 / 128$ & $1 / 256$ & $1.05 \mathrm{E}-4$ & $1.56 \mathrm{E}-8$ & $2.05 \mathrm{E} 0$ & $3.66 \mathrm{E} 0$ \\
\hline
\end{tabular}

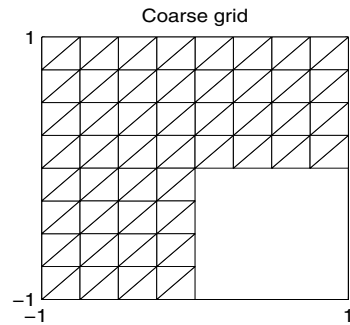

(a)

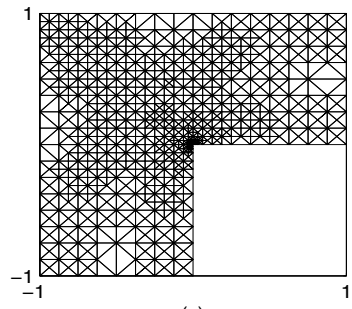

(c)



(b)

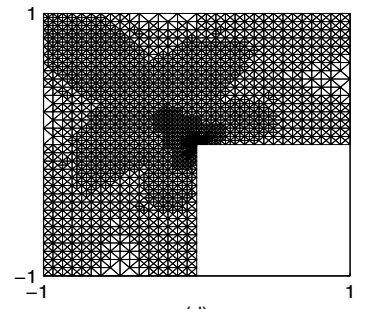

(d)

Figure 2. Coarse and fine grids for an L-shaped domain. (a) is the coarse grid. (b), (c) and (d) are the fine grids.

has singularity near the origin, and the fine grids are obtained by the adaptive local refinement procedure (see Figure 2(b), (c) and (d)). The results are shown in Table 4. Clearly, our accelerated scheme produces better approximations. 
TABLE 4. Results of Example 1 on $\Omega=(-1,1) \times(-1,1) \backslash(0,1) \times$ $(-1,0)$ based on the local refinement for the first eigenpair.

\begin{tabular}{cccccc}
\hline \hline Coarse & Fine & $\lambda_{X Z}$ & $\lambda_{A c}$ & $\lambda_{X Z}-\lambda_{h}$ & $\lambda_{A c}-\lambda_{h}$ \\
\hline \hline Fig[2 & Fig[2 & Coarse and fine grids for an L-shaped domain (see Fig. 2) \\
\hline (a) & (b) & 9.800761 & 9.761609 & $3.96 \mathrm{E}-2$ & $4.21 \mathrm{E}-4$ \\
(a) & (c) & 9.700724 & 9.698331 & $2.43 \mathrm{E}-3$ & $3.30 \mathrm{E}-5$ \\
(a) & (d) & 9.655058 & 9.654758 & $3.65 \mathrm{E}-4$ & $4.96 \mathrm{E}-6$ \\
\hline \hline$\overline{\text { Coarse }}$ & Fine & $\min _{\alpha}\left\|\nabla\left(u_{h}-\alpha u_{X Z}^{h}\right)\right\|_{L^{2}}$ & $\min _{\alpha}\left\|\nabla\left(u_{h}-\alpha u_{A c}^{h}\right)\right\|_{L^{2}}$ \\
\hline \hline Fig[2 & Fig[2 & Coarse and fine grids for an L-shaped domain (see Fig. 2) \\
\hline (a) & (b) & \multicolumn{2}{c}{$2.18 \mathrm{E}-1$} & $2.27 \mathrm{E}-2$ \\
(a) & (c) & $5.51 \mathrm{E}-2$ & $6.51 \mathrm{E}-3$ \\
(a) & (d) & $1.98 \mathrm{E}-2$ & $2.57 \mathrm{E}-3$ \\
\hline \hline
\end{tabular}

\subsection{Example 2.}

$$
\begin{aligned}
-\partial_{x x} u-\left(1+\delta^{2}\right) \partial_{y y} u & =\lambda u, \quad \text { in } \Omega, \\
u & =0, \quad \text { on } \partial \Omega,
\end{aligned}
$$

where $\Omega=(0,1) \times(0,1)$. The second and third eigenvalues are

$$
\lambda_{2}=(1+(1+\delta) / 4) \pi^{2} \text { and } \lambda_{3}=(1+\delta+1 / 4) \pi^{2} .
$$

TABLE 5. Results of Example 2 on $\Omega=(-1,1) \times(-1,1)$ for the

\begin{tabular}{|c|c|c|c|c|c|c|}
\hline Coarse & Fine & & $\lambda_{X Z}$ & $\lambda_{A c}$ & $\lambda_{X Z}-\lambda_{h, i}$ & $\lambda_{A c}-\lambda_{h, i}$ \\
\hline$\overline{\mathrm{H}}$ & $\mathrm{h}$ & \multicolumn{5}{|c|}{ Coarse and fine grids satisfying $H=h^{\frac{1}{2}}$} \\
\hline \multirow[t]{2}{*}{$1 / 4$} & $1 / 16$ & $\lambda_{2}$ & 12.593356 & 12.549865 & $5.17 \mathrm{E}-2$ & $8.21 \mathrm{E}-3$ \\
\hline & & $\lambda_{3}$ & 12.737820 & 12.695065 & $7.95 \mathrm{E}-2$ & $3.68 \mathrm{E}-2$ \\
\hline \multirow[t]{2}{*}{$1 / 16$} & $1 / 256$ & $\lambda_{2}$ & 12.338144 & 12.337866 & $2.82 \mathrm{E}-4$ & $3.23 \mathrm{E}-6$ \\
\hline & & $\lambda_{3}$ & 12.338595 & 12.338313 & $2.80 \mathrm{E}-4$ & $-2.53 \mathrm{E}-6$ \\
\hline $\mathrm{H}$ & $\mathrm{h}$ & \multicolumn{5}{|c|}{ Coarser grid (only for our accelerated scheme) $H>h^{\frac{1}{2}}$} \\
\hline \multirow[t]{2}{*}{$1 / 8$} & $1 / 256$ & $\lambda_{2}$ & - & 12.337912 & - & $4.90 \mathrm{E}-5$ \\
\hline & & $\lambda_{3}$ & - & 12.338441 & - & $1.26 \mathrm{E}-5$ \\
\hline$\overline{\mathrm{H}}$ & $\mathrm{h}$ & \multicolumn{5}{|c|}{ Coarse and fine grids satisfying $H=2 h$} \\
\hline \multirow[t]{2}{*}{$1 / 8$} & $1 / 16$ & $\lambda_{2}$ & 12.543869 & 12.541672 & $2.15 \mathrm{E}-3$ & $1.26 \mathrm{E}-5$ \\
\hline & & $\lambda_{3}$ & 12.660697 & 12.658317 & $2.41 \mathrm{E}-3$ & $3.56 \mathrm{E}-5$ \\
\hline \multirow[t]{2}{*}{$1 / 16$} & $1 / 32$ & $\lambda_{2}$ & 12.388349 & 12.388194 & $1.56 \mathrm{E}-4$ & $1.00 \mathrm{E}-7$ \\
\hline & & $\lambda_{3}$ & 12.417062 & 12.416903 & $1.60 \mathrm{E}-4$ & $1.28 \mathrm{E}-7$ \\
\hline \multirow[t]{2}{*}{$1 / 32$} & $1 / 64$ & $\lambda_{2}$ & 12.349858 & 12.349848 & $1.03 \mathrm{E}-5$ & $1.64 \mathrm{E}-7$ \\
\hline & & $\lambda_{3}$ & 12.357007 & 12.356997 & $1.02 \mathrm{E}-5$ & $8.56 \mathrm{E}-8$ \\
\hline \multirow[t]{2}{*}{$1 / 64$} & $1 / 128$ & $\lambda_{2}$ & 12.340263 & 12.340262 & $1.08 \mathrm{E}-6$ & $6.52 \mathrm{E}-7$ \\
\hline & & $\lambda_{3}$ & 12.342049 & 12.342048 & $2.16 \mathrm{E}-7$ & $-3.44 \mathrm{E}-7$ \\
\hline
\end{tabular}
second eigenvalue $\lambda_{2}=(1+(1+\delta) / 4) \pi^{2}$ and the third eigenvalue $\lambda_{3}=(1+1 / 4+\delta) \pi^{2}(\delta=1 E-5)$. 
The distance between these two eigenvalues is $\frac{3}{4} \pi^{2} \delta$. We also use the piecewise linear finite element space and choose the coarse and fine grid satisfying $H=h^{\frac{1}{2}}$, $H=2 h$ for both $\mathrm{Xu}$ and Zhou's scheme and our accelerated scheme. The coarser grid $H>h^{\frac{1}{2}}$ is used only in our accelerated scheme. The results are shown in Table 5 for $\delta=1 E-5$.

From Table 5, we can see that our scheme works better in all cases. This means our accelerated scheme is efficient and robust for eigenvalue problems which may have clustered eigenvalues.

\section{REFERENCES}

1. O. Axelsson and W. Layton, A two-level method for the discretization of nonlinear boundary value problems, SIAM J. Numer. Anal. 33 (1996), no. 6, 2359-2374. MR1427468 (98c:65181)

2. I. Babuška and J. E. Osborn, Finite element-Galerkin approximation of the eigenvalues and eigenvectors of selfadjoint problems, Math. Comp. 52 (1989), no. 186, 275-297. MR89k:65132

3. __ Eigenvalue problems, Handbook of numerical analysis, Vol. II, Handb. Numer. Anal., II, North-Holland, Amsterdam, 1991, pp. 641-787. MR.92f:65001

4. F. Chatelin, Spectral approximation of linear operators, Computer Science and Applied Mathematics, Academic Press Inc. [Harcourt Brace Jovanovich Publishers], New York, 1983. MR $86 \mathrm{~d}: 65071$

5. L Chen and C.-S. Zhang, AFEM@matlab: a Matlab package of adaptive finite element methods, Tech. report, University of Maryland at College Park, 2006.

6. C.-S. Chien and B.-W. Jeng, A two-grid discretization scheme for semilinear elliptic eigenvalue problems, SIAM J. Sci. Comput. 27 (2006), no. 4, 1287-1304. MR2006j:65330

7. C.N. Dawson and M.F. Wheeler, Two-grid methods for mixed finite element approximations of nonlinear parabolic equations, Domain decomposition methods in scientific and engineering computing (University Park, PA, 1993), Contemp. Math., vol. 180, Amer. Math. Soc., Providence, RI, 1994, pp. 191-203. MR95j:65117

8. C.N. Dawson, M.F. Wheeler, and C.S. Woodward, A two-grid finite difference scheme for nonlinear parabolic equations, SIAM J. Numer. Anal. 35 (1998), no. 2, 435-452. MR99b:65097

9. G.H. Golub and C.F. Van Loan, Matrix computations, Johns Hopkins Univ. Press, 1996. MR $1417720(97 \mathrm{~g}: 65006)$

10. J. Jin, S. Shu, and J. Xu, A two-grid discretization method for decoupling systems of partial differential equations, Math. Comp. 75 (2006), no. 256, 1617-1626. MR.2007e:65121

11. W. Layton and W. Lenferink, Two-level Picard and modified Picard methods for the NavierStokes equations, Appl. Math. Comput. 69 (1995), no. 2-3, 263-274. MR95m:65191

12. W. Layton and L. Tobiska, A two-level method with backtracking for the Navier-Stokes equations, SIAM J. Numer. Anal. 35 (1998), no. 5, 2035-2054. MR.99g:65115

13. M. Marion and J. Xu, Error estimates on a new nonlinear Galerkin method based on two-grid finite elements, SIAM J. Numer. Anal. 32 (1995), no. 4, 1170-1184. MR96f:65136

14. B.N. Parlett, The symmetric eigenvalue problem, Society for Industrial Mathematics, 1998. MR.1490034 (99c:65072)

15. G. Peters and J.H. Wilkinson, Inverse iteration, ill-conditioned equations and Newton's method, SIAM review 21 (1979), no. 3, 339-360. MR.535118 (80f:65040)

16. T. Utnes, Two-grid finite element formulations of the incompressible Navier-Stokes equations, Comm. Numer. Methods Engrg. 13 (1997), no. 8, 675-684. MR98d:76110

17. J. Xu, A new class of iterative methods for nonselfadjoint or indefinite problems, SIAM J. Numer. Anal. 29 (1992), no. 2, 303-319. MR92k:65063

18. , A novel two-grid method for semilinear elliptic equations, SIAM J. Sci. Comput. 15 (1994), no. 1, 231-237. MR94m:65178

19. , Two-grid discretization techniques for linear and nonlinear PDEs, SIAM J. Numer. Anal. 33 (1996), no. 5, 1759-1777. MR97i:65169

20. J. Xu and A. Zhou, Local and parallel finite element algorithms based on two-grid discretizations, Math. Comp. 69 (2000), no. 231, 881-909. MR2000j:65102

21. _ Local and parallel finite element algorithms based on two-grid discretizations for nonlinear problems, Adv. Comput. Math. 14 (2001), no. 4, 293-327. MR2002h:65198 
22. _ـ A two-grid discretization scheme for eigenvalue problems, Math. Comp. 70 (2001), no. 233, 17-25. MR2001f:65130

23. _ Local and parallel finite element algorithms for eigenvalue problems, Acta Math. Appl. Sin. Engl. Ser. 18 (2002), no. 2, 185-200. MR.2004m:65184

Department of Mathematics, Zhejiang University, Yuquan Campus, Hangzhou, 310027, People's Republic of ChinA

E-mail address: huxiaozhezju@gmail.com

Department of Mathematics, Zhejiang University, Yuquan Campus, Hangzhou, 310027, People's Republic of ChinA

E-mail address: xiaoliangcheng@zju.edu.cn 